\title{
Ação do fungo Beauveria bassiana (Balsamo) Vuillemin, 1912 sobre a fase parasitária do carrapato Anocentor nitens (Neumann, 1897) Schulze, 1937 (Acari: ixodidae)
}

\author{
Action of the fungus Beauveria bassiana (Balsamo) Vuillemin, \\ 1912 on the parasitic phaseof the tick Anocentor nitens \\ (Neumann, 1897) Schulze, 1937 (Acari: ixodidae) \\ Silvia Gonzalez Monteiro ${ }^{1}$ Thiago Campanharo Bahiense ${ }^{2}$ \\ Vânia Rita Elias Pinheiro Bittencourt ${ }^{3}$
}

\section{RESUMO}

A patogenicidade do fungo Beauveria bassiana (isolado 986) foi avaliada no desenvolvimento da fase parasitária do Anocentor nitens, acompanhando o desenvolvimento de larvas não alimentadas até o estágio adulto. Foram utilizados dois tratamentos e um grupo controle com cinco repetições cada. No tratamento 1 (T1), as orelhas dos bovinos foram banhadas com a suspensão fúngica de $10^{8}$ conídios $\mathrm{ml}^{-1}$; no tratamento 2 (T2), as orelhas foram banhadas com água destilada e espalhante adesivo. No grupo controle (C), as orelhas foram banhadas com água destilada. Após 24 horas dos tratamentos, as orelhas de cada animal foram infestadas com $100 \mathrm{mg}$ de larvas. Os parâmetros analisados, em cada tratamento, foram: número e peso de fêmeas recuperadas; periodos de pré-postura, postura, incubação, eclosão e longevidade; percentual de eclosão; peso da massa de ovos e da quenógina; indices de eficiência reprodutiva e nutricional. Verificou-se que, no T1 e T2, o número de fêmeas recuperadas, após 35 dias, foi significantemente menor $(P \leq 0,05)$ que no $C$. $O$ número de fêmeas recuperadas foi 35, 70 e 117 fêmeas, no T1, T2 e C, respectivamente. As fêmeas oriundas das larvas sobreviventes dos grupos tratados não sofreram ação deletéria do entomopatógeno avaliado.

Palavras-chave: Anocentor nitens, Beauveria bassiana, fungo entomopatogênico, controle microbiano.

\section{ABSTRACT}

This study evaluated the pathogenicity of the fungus Beauveria bassiana (Isolate 986) on the development of the parasitic phase of Anocentor nitens by accompanying the development of unfed larvae until the adult stage. Two treated and one control group were used with five repetitions each. In the first treated group (T1), cattle ears were bathed with a fungal suspension of $10^{8}$ conidia $\mathrm{ml}^{-1}$, second treated group (T2), the ears were bathed with distilled water and Tween 80 $1 \%$ and the control group $(C)$, the ears were bathed with distilled water. One day after treatment, the ears of each animal were infested with $100 \mathrm{mg}$ of larvae. The parameters analyzed in each treatment were: number and weight of females recovered; periods of pre-oviposition, oviposition, incubation, eclosion and longevity; percentage of eggs hatched; weight of the egg mass and of females after oviposition. We found that in the two treated groups the number of females recovered was significantly lower $(P \leq 0.05)$ than in the control group. 35, 70 and 117 females were recovered from the $T 1, T 2$ and $C$, respectively. The females obtained from the surviving larvae of the treated groups did not suffer any deleterious effects from the entomopathogen studied.

Key words: Anocentor nitens, Beauveria bassiana, entomopathogenic fungus, microbial control

\section{INTRODUÇ̃̃o}

O carrapato Anocentor nitens é um ectoparasita encontrado parasitando principalmente o conduto auditivo de equídeos (ARAGÃO \& FONSECA, 1961). A sua presença provoca irritação no

${ }^{1}$ Médico Veterinário, Doutor, Universidade Federal de Santa Maria.

${ }^{2}$ Médico Veterinário, Mestrando Curso de Pós-graduação em Ciência Veterinária, Universidade Federal Rural do Rio de Janeiro (UFRRJ).

${ }^{3}$ Médico Veterinário, PhD, Departamento de Parasitologia Animal, IV, UFRRJ, BR 465 Km 7, 23890-000, Seropédica, RJ. Email:vaniabit@ufrj.br. Autor para correspondência. 
hospedeiro além de servir como agente transmissor de uma variedade de agentes patogênicos causadores de enfermidades como babesiose e anemia infecciosa eqüina (PFEIFER et al., 1995). Além disso, as lesões na pele, ocasionadas pela sua presença, podem servir de porta de entrada a infecções bacterianas secundárias e miíases.

O fungo Beauveria bassiana (Balsamo) Vuillemin 1912 pertence à classe Hyphomycetes, família Moniliaceae, e é comumente encontrado no solo. É uma das espécies mais estudadas no controle de artrópodes, provavelmente em função da ampla distribuição geográfica e da variedade de seus hospedeiros (ALVES,1998). A maioria dos trabalhos nos quais esse fungo foi utilizado objetiva o controle de pragas agrícolas, mas alguns autores já demonstraram bons resultados no uso de B. bassiana em controle de parasitos de animais. BITTENCOURT et al. (1996) verificaram a patogenicidade in vitro do fungo B. bassiana (isolados 986 e 747) em ovos e larvas não alimentadas de Boophilus microplus e observaram uma elevada diminuição do percentual de eclosão e também maior taxa de mortalidade das larvas nos grupos tratados, concluindo que esse fungo tem uma elevada capacidade carrapaticida.

O uso de suspensões de $\boldsymbol{B}$. bassiana, em ovos e em larvas de Rhipicephalus sanguineus, diminuiu o percentual de eclosão e sobrevivência de larvas conforme aumentava-se a concentração dos conídios (MONTEIRO et al., 1998a e 1998b).

BITTENCOURT et al. (1999) também utilizaram suspensões de B. bassiana e Metarhizium anisopliae em larvas de Anocentor nitens e constataram que a média de mortalidade das larvas tratadas com a suspensão quantificada em $10^{8}$ conídios/ $\mathrm{ml}$ foi sempre superior a $80 \%$, enquanto no grupo controle não excedeu a $1 \%$, fato este que comprova a eficiência do fungo para o controle deste carrapato. Outro trabalho que também demonstra a patogenicidade dos fungos entomopatogênicos sobre o carrapato $\boldsymbol{A}$. nitens é o de BITTENCOURT et al. (2000) que testaram três isolados de M. anisopliae e um de B. bassiana em ovos de $\boldsymbol{A}$. nitens e constataram uma diminuição na eclosão de larvas oriundas de ovos tratados com os isolados em relação ao grupo controle (30 e 90\% respectivamente).

KAAYA et al. (1996) evidenciaram, em testes com B. bassiana e M. anisopliae in vitro, que a concentração $10^{8}$ conídios $/ \mathrm{ml}$ inibiu a eclosão de larvas de Amblyomma variegatum oriundas de fêmeas ingurgitadas tratadas; os ovos de fêmeas tratadas com M. anisopliae tiveram um índice de eclosão de larvas de $3,9 \%$ e das tratadas com B. bassiana não houve eclosão, o que revelou uma grande redução se comparado ao grupo controle no qual o índice de eclosão larval foi de $68,3 \%$.

MONTEIRO (2000) procedeu avaliação in vitro do isolado 986 de $\boldsymbol{B}$. bassiana sobre ovos, larvas e fêmeas ingurgitadas de $\boldsymbol{A}$. nitens e concluiu que o estágio de larva não alimentada foi o mais susceptível, obtendo, para este estágio, uma CL 50 de 5,09 X $10^{3} \mathrm{e}$ uma CL90 de 4,9 X $10^{6}$ conídios $/ \mathrm{ml}$.

Portanto, o objetivo deste trabalho foi avaliar o efeito in vivo do isolado 986 do fungo $\boldsymbol{B}$. bassiana quando aplicado sobre larvas do carrapato A. nitens, no desenvolvimento da sua fase parasitária, utilizando bovinos como hospedeiros, em condições de estábulo.

\section{MATERIAL EMÉTODOS}

O experimento foi desenvolvido na Estação Experimental para Pesquisas Parasitológicas W. O Neitz da Universidade Federal Rural do Rio de Janeiro, Brasil, no período de março de 1997 a outubro de 1998.

Foram utilizados 15 bovinos machos, 3/4 holandês com \pm 18 meses de idade e peso médio de $300 \mathrm{~kg}$, que foram mantidos em baias individuais, onde receberam ração comercial, capim picado, sal mineral e água à vontade diariamente. Os animais foram tratados com anti-helmínticos (fenbendazole), carrapaticidas (amitraz) e babesicidas (diamidina). As baias individuais também foram lavadas com carrapaticida antes da chegada dos animais. Uma semana após o banho carrapaticida, fez-se uma lavagem em todo o corpo do animal com água e sabão neutro para retirada do excesso do produto. Após 30 dias, cada animal teve um saco de pano fixado na base das orelhas com cola, que foi fechado com velcro na extremidade lateral e superior.

As larvas utilizadas para a infestação foram obtidas do laboratório de Ixodologia da UFRRJ. Para a infestação, os ovos foram pesados em cotas de 100mg (1740 larvas) cada, colocados em seringas descartáveis cortadas na extremidade superior e levados à câmara climatizada $\left(27^{\circ} \mathrm{C}\right.$ e $\geq 80 \%$ UR) para eclosão das larvas. Após o final da eclosão esperouse mais 20 dias para completa quitinização e fez-se a infestação dos animais.

$\mathrm{O}$ isolado do fungo foi cedido pelo Departamento de Entomologia da Escola Superior de Agricultura Luiz de Queiroz da USP. Esse isolado foi repicado em meio de arroz (BITTENCOURT et al., 1992) e, após a obtenção dos conídios, foi feita uma suspensão de $10^{8}$ conídios $/ \mathrm{ml}$ quantificada em câmara de Neubauer (ALVES, 1998). Os conídios foram suspensos numa solução de espalhante adesivo (Tween 80 a 1\%). 
Os animais foram divididos em três tratamentos, dois tratados e um controle com cinco repetições em cada tratamento. No tratamento 1 (T1), as orelhas dos bovinos foram banhadas com a suspensão fúngica de $10^{8}$ conídios $\mathrm{ml}^{-1}$, e após 24 horas, foram infestadas com $100 \mathrm{mg}$ de larvas não alimentadas. No tratamento 2 (T2), as orelhas foram banhadas com água destilada e espalhante adesivo(Tween 80 a 1\%) e, após 24 horas, foram colocadas $100 \mathrm{mg}$ de larvas. No grupo controle (C), as orelhas foram banhadas com água destilada e, após 24 horas, foram infestadas com $100 \mathrm{mg}$ de larvas.

Todas as fêmeas oriundas deste experimento foram recuperadas após no máximo 35 dias e levadas ao laboratório para a análise dos seguintes parâmetros: número de fêmeas ingurgitadas recuperadas em cada tratamento e o \% de controle, peso das fêmeas recuperadas, períodos de pré-postura, postura, incubação, eclosão e longevidade, peso da massa de ovos, percentual de eclosão, peso da quenógina, índices de eficiência reprodutiva e nutricional (BENNETT, 1974). Para a análise dos dados obtidos, foi feita análise de variância (ANOVA) para saber se houve variações entre os tratamentos. A ANOVA foi seguida por aplicação do teste de Tukey para comparação entre as médias calculando-se o coeficiente de variação para verificar a precisão dos dados.

\section{RESULTADOS}

No T1, no qual se utilizou a suspensão de B. bassiana, o número de fêmeas recuperadas foi de 35 ; no T 2 recuperaram-se 70 fêmeas, enquanto que, no $\mathrm{C}$, a recuperação foi de 117 fêmeas. O percentual de controle obtido para o $\mathrm{T} 1$ foi de $70,1 \%$ e para o $\mathrm{T} 2$ foi de $32,5 \%$. O peso médio das fêmeas ingurgitadas recuperadas teve uma variação de 260 a $315 \mathrm{mg}$, sendo que no $\mathrm{T} 1$, o peso foi superior aos outros tratamentos. Os parâmetros relativos aos parâmetros biológicos observados podem ser verificados na Tabela 1 .

\section{DISCUSSÃO}

O bioensaio in vivo, com larvas em bovinos, demonstrou uma grande diferença entre o número médio de fêmeas recuperadas no grupo controle comparado aos tratamentos, o que indica que houve uma redução significativa no número de larvas no tratamento 1 , fator que refletiu no número total de fêmeas ingurgitadas coletadas. CASTRO et al. (1997) obteve um percentual de controle de $64,8 \%$ em testes de estábulo com o carrapato B. microplus utilizando uma suspensão de $10^{8}$ conídios/ml do fungo $\boldsymbol{M}$. anisopliae, resultados estes similares ao do presente trabalho, que foi de $70,1 \%$ no T1. Os dados obtidos com o peso das fêmeas recuperadas, nos grupos tratados foram superiores ao grupo controle, fato que deve ter ocorrido por falta de competição entre os carrapatos no pavilhão auricular. Como já sugerido por SUTHERST et al. (1973), uma menor quantidade de larvas na infestação do animal, (que no presente trabalho foi em função do efeito patogênico do entomopatógeno) gera uma menor competição entre elas, podendo levar a resultados melhores com relação ao peso de fêmeas quando ingurgitadas. SILVA et al. (1996) também verificaram resultados semelhantes aos do presente trabalho e também ao trabalho supracitado, o que reforça as afirmações feitas neste trabalho e, como conseqüência, todos os parâmetros relacionados ao peso inicial das fêmeas (massa de ovos, peso da quenógina, IEN) seriam afetados.

Os dados obtidos nos parâmetros peso de fêmeas recuperadas, período de postura, peso da massa de ovos, peso da quenógina e índice de eficiência nutricional, avaliados na tabela 1 , demonstraram diferenças significativas entre $\mathrm{T} 2$ ou $\mathrm{C}$ e o T1. Apesar desses dados, é possível que estes resultados não tenham relação com o efeito patogênico do entomopatógeno, pois todos os demais parâmetros observados neste bioensaio, a partir de larvas que sobreviveram ao tratamento, levam a crer que houve interferência do tamanho da população na infestação dos pavilhões auditivos, propiciando resultados favoráveis nos parâmetros biológicos dos grupos tratados, que por sua vez, apresentaram menor número de fêmeas sobreviventes. SUTHERST et al. (1973) afirmam que ocorre competição intra-específica na espécie B. microplus, e que essa competição é dependente da densidade da infestação.

Para o período de postura, DUNN et al. (1915) e DRUMMOND et al. (1969) obtiveram 16 e 14,5 dias de postura, respectivamente, em seus trabalhos com biologia de $\boldsymbol{A}$. nitens, dados que são semelhantes aos do presente trabalho e que podem indicar que a geração de fêmeas obtidas, a partir de larvas infectadas, não foram susceptíveis ao isolado. Os trabalhos de SUTHERST et al. (1973) sobre a densidade - mortalidade dependente, podem explicar estes achados. SILVA et al. (1996), trabalhando com o B. microplus em coelhos ,também verificaram que as infestações realizadas com número menor de larvas produziram fêmeas ingurgitadas mais pesadas e, conseqüentemente, com uma massa de ovos maior, afirmando que, em menor número, os indivíduos não precisaram competir pelo espaço disponível no pavilhão auricular dos coelhos, permitindo ainda a procura de um local de eleição para 
Tabela 1 - Média dos principais parâmetros biológicos de fêmeas de Anocentor nitens coletadas em teste de estábulo em bovinos artificialmente infestados e tratados com uma suspensão de Beauveria bassiana.

\begin{tabular}{|c|c|c|c|c|c|c|c|c|c|c|c|c|}
\hline $\begin{array}{l}\text { Trata- } \\
\text { mentos }\end{array}$ & & $\begin{array}{l}\text { PFR } \\
(\mathrm{mg})\end{array}$ & $\begin{array}{c}\text { PPP } \\
\text { (dias) }\end{array}$ & $\begin{array}{c}\text { PP } \\
\text { (dias) }\end{array}$ & $\begin{array}{l}\text { PMO } \\
(\mathrm{mg})\end{array}$ & $\begin{array}{c}\text { PQ } \\
(\mathrm{mg})\end{array}$ & $\begin{array}{l}\text { IER } \\
(\%)\end{array}$ & $\begin{array}{l}\text { IEN } \\
(\%)\end{array}$ & $\begin{array}{c}\text { PM } \\
\text { (dias) }\end{array}$ & $\begin{array}{c}\text { PI } \\
\text { (dias) }\end{array}$ & $\begin{array}{c}\mathrm{PE} \\
\text { (dias) }\end{array}$ & $\begin{array}{l}\mathrm{EC} \\
(\%) \\
\end{array}$ \\
\hline \multirow[t]{2}{*}{1} & 凶 & $315^{\mathrm{a}}$ & $3,5^{\mathrm{a}}$ & $15,1^{\mathrm{a}}$ & $177^{\mathrm{a}}$ & $64,2^{\mathrm{a}}$ & $52,3^{\mathrm{a}}$ & $65,7^{\mathrm{a}}$ & $23,6^{\mathrm{a}}$ & $24,7^{\mathrm{a}}$ & $3,9^{\mathrm{a}}$ & $29,2^{\mathrm{a}}$ \\
\hline & S & 112,1 & 1,0 & 3,8 & 64,2 & 31,7 & 11,1 & 13,2 & 5,9 & 1,9 & 2,0 & 23,5 \\
\hline \multirow[t]{2}{*}{2} & 凶 & $260^{\mathrm{b}}$ & $3,5^{\mathrm{a}}$ & $12,5^{\mathrm{b}}$ & $149^{\mathrm{b}}$ & $59,0^{\mathrm{b}}$ & $51,4^{\mathrm{a}}$ & $63,5^{b}$ & $21,2^{\mathrm{a}}$ & $25,7^{\mathrm{a}}$ & $3,5^{\mathrm{a}}$ & $36,5^{\mathrm{a}}$ \\
\hline & $\mathrm{S}$ & 95,3 & 1,2 & 2,8 & 53,7 & 35,1 & 10,8 & 10,6 & 5,2 & 2,0 & 1,4 & 27,3 \\
\hline \multirow[t]{2}{*}{ Controle } & 凶 & $278^{\mathrm{b}}$ & $3,8^{\mathrm{a}}$ & $12,2^{\mathrm{b}}$ & $142^{\mathrm{b}}$ & $41,6^{\mathrm{b}}$ & $50,6^{\mathrm{a}}$ & $60,5^{b}$ & $22,1^{\mathrm{a}}$ & $26,4^{\mathrm{a}}$ & $3,4^{\mathrm{a}}$ & $31,7^{\mathrm{a}}$ \\
\hline & $\mathrm{S}$ & 119,2 & 1,2 & 3,9 & 75,6 & 22,5 & 15,2 & 16,9 & 5,5 & 2,1 & 1,8 & 20,7 \\
\hline
\end{tabular}

Médias seguidas da mesma letra na coluna não diferem entre si $(\mathrm{p} \geq 0,05)$

PFR - Peso fêmeas recuperadas; $\quad$ PPP - Período pré postura; $\quad$ PP-Período postura; $\quad$ PMO - Peso massa de ovos; PQ - Peso da quenógina;

IER - Índice de eficiência reprodutiva; IEN - Índice de eficiência nutricional; $\quad$ PM - Período de mortalidade de fêmeas; $\quad$ PI Período de incubação dos ovos; PE - Período de eclosão das larvas; $\quad$ EC - \% Eclosão;

\- média; $\mathrm{S}$-desvio padrão.

fixação. Esses mesmos autores também encontraram um IEN muito superior quando infestaram coelhos com uma quantidade menor de larvas, o que demonstra mais uma vez que o efeito da baixa competitividade intraespecífica (causada por uma infestação mais baixa em decorrência da mortalidade gerada pelo entomopatógeno) pode justificar os resultados encontrados no presente trabalho.

Todos os dados obtidos, no grupo controle, foram similares aos encontrados por SANAVRIA et al. (1996), BORGES et al. (1993) e DESPINS (1992) em trabalhos realizados sob as mesmas condições para estudo da biologia desta espécie de carrapato.

Os resultados obtidos no presente trabalho e no trabalhode outros autores já citados demonstram que fungos entomopatogênicos podem desempenhar papel importante no controle de carrapatos. Os países de clima quente e úmido são propícios ao desenvolvimento não apenas de fungos, como também de outros entomopatógenos, e a busca de formas de controle alternativo deve continuar. Os agentes de controle biológico que afetam os ixodídeos e sua ação patogênica ainda são muito pouco estudados. Maiores informações sobre as doenças que afetam os carrapatos, sua susceptibilidade aos entomopatógenos e sobre o funcionamento do seu sistema imune são necessárias para a formação de uma base para a aplicação eficaz do controle biológico. Além disso, a busca de formulações deste entomopatógeno, de modo a propiciar melhores condições ao fungo, aumentando seu efeito patogênico a campo e o seu uso em programas de manejo integrado, devem ser pesquisados.

\section{REFERÊNCIAS BIBLIOGRÁFICAS}

ALVES, S.B. (Coord.). Controle microbiano de insetos. Piracicaba, SP : FEALQ, 1998. 891p.

ARAGÃO, H.; FONSECA, F. Notas de ixodologia. VIII. Lista e chave para os representantes da fauna ixodológica brasileira. Memórias do Instituto Oswaldo Cruz, v.59, n.2, p.115$129,1961$.

BENNETT, G.F. Oviposition of Boophilus microplus (Canestrini)(Acarida: Ixodidae). I. Influence of tick size on egg production. Acarologia, v.16, n.1, p.52-61, 1974.

BITTENCOURT, V.R.E.P.; MASSARD, C.L.; LIMA, A.F. Ação do fungo Metarhizium anisopliae (Metschnikoff, 1879) Sorokin, 1883, sobre o carrapato Boophilus microplus (Canestrini, 1887). Arquivos da Universidade Federal Rural do Rio de Janeiro, v.15, n.2, p.197-202, 1992.

BITTENCOURT, V. R. E. P. et al. Avaliação dos efeitos do contato de Beauveria bassiana (Balsamo) Vuillemin com ovos e larvas de Boophilus microplus (Canestrini, 1887) (Acari: Ixodidae). Revista Brasileira de Parasitologia Veterinária, v.5, n.2, p.81-84, 1996.

BITTENCOURT, V.R.E.P. et al. Ação in vitro do Metarhizium anisopliae (Metschnikoff, 1879) Sorokin, 1883 e Beauveria bassiana (Balsamo) Vuillemin, 1912 sobre ovos do carrapato Anocentor nitens (Neumann, 1897) (Acari: Ixodidae). Revista Brasileira de Medicina Veterinária, v.22, n.6, p.248-251, 2000 .

BITTENCOURT, V.R.E.P. et al. Ação dos fungos Beauveria bassiana (Balsamo) Vuillemin, 1912 e Metarhizium anisopliae (Metschnikoff, 1879) Sorokin, 1883 sobre larvas do carrapato Anocentor nitens (Acari: Ixodidae). Parasitologia al dia , v.23, n.3-4, p.82-86, 1999.

BORGES, L.M.F.; LEITE, R.C. Aspectos biológicos do Dermacentor nitens (Neumann, 1897) em condições de 
laboratório. Arquivo Brasileiro de Medicina Veterinária e Zootecnia., v.45, n.6, p.585-591, 1993.

CASTRO, A.B.A. et al. Eficácia in vivo do fungo Metarhizium anisopliae (isolado 959) sobre o carrapato Boophilus microplus em teste de estábulo. Revista Universidade Rural - Série Ciências da Vida, v.19, n.1-2, p.73-82, 1997.

DESPINS, J.L. Effects of temperature and humidity on ovipositional biology and egg development of the tropical horse tick, Dermacentor (Anocentor) nitens. Journal of Medical Entomology, v.29, n.2, p.332-337, 1992.

DRUMMOND, R.O. et al. Laboratory study of Anocentor nitens (Neumann) (Acarina: Ixodidae), the tropical horse tick. Journal of Medical Entomology, v.6, p.150-154, 1969.

DUNN, L.H. Observations on the preoviposition and incubation periods of Dermacentor nitens in Panama (Arach., Acar.). Entomological News, v.26, p.214-219, 1915.

KAAYA, G.P.; MWANGI, E.N.; OUNA, E.A. Prospects for Biological Control of Livestock Ticks, Rhipicephalus appendiculatus and Amblyomma variegatum, Using the Entomogenous Fungi Beauveria bassiana and Metarhizium anisopliae. Journal of Invertebrate Pathology, v.67, p.1520, 1996.

MONTEIRO, S.G. Ação do isolado 986 do fungo Beauveria bassiana (Balsamo) Vuillemin, 1912, sobre o carrapato Anocentor nitens (Neumann) Schulze, 1937. 2000. $75 \mathrm{f}$.
Tese (Doutorado em Parasitologia Veterinária) - Universidade Federal Rural do Rio de Janeiro.

MONTEIRO, S. G. et al. Efeito dos fungos entomopatogênicos Metarhizium anisopliae e Beauveria bassiana em ovos de Rhipicephalus sanguineus (Acari: Ixodidae). Ciência Rural, v.28, n.3, p.351-354, 1998a.

MONTEIRO, S.G. et al. Pathogenicity under laboratory conditions of the fungi Beauveria bassiana and Metarhizium anisopliae on larvae of the tick Rhipicephalus sanguineus (Acari: Ixodidae). Revista Brasileira de Parasitologia Veterinária, v.7, n.2, p.113-116, 1998 b.

PFEIFER, B.I. et al. Epidemiological aspects of equine babesioses in a herd of horses in Brazil. Veterinary Parasitology, v.58, n.1-2, p.1-8, 1995.

SANAVRIA, A.; PRATA, M.C.A.; MORAIS, M.C. Determinação de alguns parâmetros biológicos de Anocentor nitens (Neumann, 1897) (Acari: Ixodidae). Arquivos da Faculdade de Veterinária da UFRGS, v.24, n.2, p.87-94, 1996.

SILVA, C.L.G. et al. Efeito do número de larvas de Boophilus microplus (Can., 1887) (Acari: Ixodidae) sobre a recuperação de fêmeas ingurgitadas em coelhos. Revista Brasileira de Ciência Veterinária., v.3, n.2, p.59-61, 1996.

SUTHERST, R.W. et al. Intraespecific competition of Boophilus microplus (Canestrini) on cattle. Journal of Applied Ecology, v.10, p.855-862, 1973. 